\title{
ReaR

\section{Driving pressure y mortalidad en el síndrome de distrés respiratorio agudo}

Artículo original: Aoyama H, Pettenuzzo T, Aoyama K, Pinto R, Englesakis M, et al. Association of Driving Pressure With Mortality Among Ventilated Patients With Acute Respiratory Distress Syndrome: A Systematic Review and Meta-Analysis. Crit Care Med. 2018 Feb;46(2):300-306. doi: 10.1097/CCM.0000000000002838. ( PubMed)

de la Calle Gil I, Navarro Casado R, García Álvarez R

Hospital Universitario 12 de Octubre. Madrid.

\section{Resumen}

Las actuales guías de manejo del síndrome de distrés respiratorio agudo (SDRA) recomiendan una ventilación protectora: volumen corriente bajo, presión positiva al final de la espiración (PEEP) adecuada y maniobras de reclutamiento alveolar. Sin embargo, estudios recientes han mostrado que la driving pressure podría ser la variable que mejor se correlaciona con la supervivencia en pacientes con SDRA.

\section{Introducción}

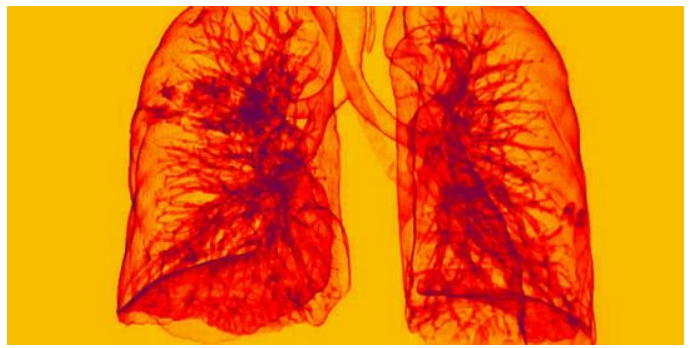

Las actuales guías de manejo del síndrome de distrés respiratorio agudo (SDRA) recomiendan una ventilación protectora: volumen corriente bajo, presión positiva al final de la espiración (PEEP) adecuada y maniobras de reclutamiento alveolar. Sin embargo, estudios recientes han mostrado que la driving pressure podría ser la variable que mejor se correlaciona con la supervivencia en pacientes con SDRA.

El síndrome de distrés respiratorio agudo (SDRA) es una entidad frecuente en las unidades de cuidados intensivos, representando el $10.4 \%$ de los ingresos $\mathrm{y}$ el $23.4 \%$ del total de pacientes que requieren ventilación mecánica invasiva (VM) durante su estancia en UCI (Unidad de Cuidados Intensivos). Debido a su etiología multifactorial, muchas veces es una patología infradiagnosticada y con una morbimortalidad importante ${ }^{2}$. Las actuales recomendaciones acerca de su manejo ventilatorio se resumen en una ventilación protectora, entendida como volumen tidal bajo, PEEP (presión positiva al final de la espiración óptima), presión plateau baja $\mathrm{y}$ maniobras de reclutamiento.

La driving pressure o delta de presión (DP), definida como la diferencia entre la presión teleinspiratoria o plateau y la PEEP, podría ser la variable más fuertemente asociada con mortalidad en el SDRA.

\section{Material y Métodos}

El artículo original es una revisión sistemática cuyo objetivo principal es determinar la asociación entre DP y mortalidad en pacientes con SDRA y 
ventilación mecánica. Como objetivos secundarios destacar la identificación de una DP concreta y la comparación de la DP con la presión plateau y la complianza respiratoria como predictores de mortalidad.

Se incluyeron análisis controlados randomizados, de cohortes, y de casos y controles en pacientes adultos con SDRA y VM. Estos trabajos compararon diferentes niveles de DP o bien la DP con otros parámetros respiratorios. Se encontraron 16 estudios potenciales, de los cuales solo 7 cumplieron los criterios de inclusión. Dos de ellos provienen del mismo artículo original, de modo que los datos se trataron como si fueran un único análisis. Se obtuvo una población total de 6.062 pacientes.

La variable principal a analizar fue la mortalidad hospitalaria. Como variables secundarias se incluyeron tiempo de estancia en UVI, días de VM, complicaciones asociadas a la VM (barotrauma, neumonía), tratamientos adyuvantes del SDRA (perfusión de relajantes neuromusculares, óxido nítrico, decúbito prono, ECMO) $\mathrm{y}$ medidas fisiológicas (oxigenación, complianza, presión plateau, PEEP, volumen tidal), fracaso multiorgánico.

\section{Resultados}

Todas las publicaciones incluidas demostraron una asociación entre mayor DP y la mortalidad en el SDRA. Asimismo, todos estudiaron la asociación entre mayor y menor DP con mortalidad (excepto un análisis que se basó en la DP transpulmonar, es decir, sin la complianza de la pared torácica). Sin embargo, cada uno de ellos empleó diferentes puntos de corte de DP para cada grupo (alta frente a baja) según las medianas de sus cohortes. La mediana de todos ellos fue $15 \mathrm{~cm}$ de $\mathrm{H}_{2} \mathrm{O}$.
En el metaanálisis de cuatro de los seis manuscritos incluidos se asoció de manera estadísticamente significativa (RR 1.44, 95\% CI 1.11-1.88; I2=85\%) mayores niveles de DP con mortalidad entre pacientes con SDRA y VM. Se excluyeron dos de ellos porque no comparaban entre sí DP alta frente a baja y porque no registraban eventos adversos (mortalidad), respectivamente.

No se pudieron hacer análisis secundarios, como el riesgo de barotrauma asociado a DP, por falta de datos.

\section{Discusión}

La DP no sólo es la diferencia entre presión plateau y PEEP, si no que representa también la relación existente entre el volumen corriente/tidal y la complianza estática del sistema respiratorio, representando el estrés aplicado a los pulmones. La importancia de la DP ya fue propuesta en 1998 por el equipo de Amato $^{4}$ y casi veinte años más tarde el mismo equipo asoció DP y mortalidad en pacientes con SDRA ${ }^{5}$. Sin embargo, su carácter retrospectivo y la ausencia de ensayos no nos permite determinar si esta estrategia (limitar la DP) es superior a la actual y cuál sería el valor de la DP objetivo.

Tras una búsqueda exhaustiva, el metaanálisis incluye solo siete trabajos no observacionales (aunque estos con una población a analizar grande) y ningún ensayo clínico. Otro punto débil del mismo es la ausencia de uniformidad en la escala de severidad entre las publicaciones. Sólo 4 de los 7 incluidos investigaron otras variables de mecánica respiratoria (por ejemplo, complianza y elastancia). Tampoco dilucida qué es superior para predecir mortalidad: DP o presión plateau.

La determinación de un punto de corte de DP no queda clara. Tampoco la 
estrategia óptima para conseguir una menor DP, es decir, los parámetros respiratorios a modificar y hacia qué sentido (mayor o menor PEEP, volumen tidal, etc.)

Por lo que respecta a la utilidad de la DP transpulmonar (DP sin la complianza de la pared torácica), no queda clara. Sólo un estudio aplica este concepto y concluye que ambas DP tienden a ser similares.

Como puntos fuertes del metaanálisis cabe destacar que todos los trabajos demostraron la asociación entre DP elevada y mayor mortalidad (aunque cada uno con un valor de corte de DP para cada grupo: bajo versus alto) y la población a analizar fue considerable (más de 6.000 pacientes). El valor medio de DP es de $15 \mathrm{~cm}$ de $\mathrm{H}_{2} 0$, lo que sugiere que el objetivo podría estar entre 13-15 mm de $\mathrm{Hg}$. Este podría ser el punto de partida para investigaciones futuras.

Asimismo, constituye el primer metaanálisis de DP en pacientes con SDRA y VM, que confirma lo que ya se determinó en otros análisis individuales respecto a la asociación entre DP elevada y mayor mortalidad.

\section{Conclusiones}

La DP elevada se asocia a una mayor mortalidad en pacientes con SDRA y VM. Sin embargo, son necesarios ensayos clínicos prospectivos que evalúen la eficacia y seguridad de la ventilación basada en la DP comparada con el actual estándar (ventilación protectora). No existe evidencia suficiente para recomendar un valor concreto de DP. Actualmente hay al menos tres ensayos clínicos en marcha de DP en SDRA registrados en clinicaltrials.gov. Está por concretar si la DP podría ser un marcador de severidad del SDRA, un límite de seguridad o un objetivo en sí mismo dentro de la estrategia de ventilación protectora.

\section{Bibliografía}

1.- Hiroko Aoyama, MD, MScCH; Tommaso Pettenuzzo, MD; Kazuyoshi Aoyama, MD, PhD(c), EDIC; Ruxandra Pinto, PhD; Marina Englesakis, MLIS; Eddy Fan, MD, PhD, FRCPC. Association of Driving Pressure With Mortality Among Ventilated Patients With Acute Respiratory Distress Syndrome: A Systematic Review and Meta-Analysis. Critical Care Medicine Journal 2018; 46(2):300-306. (HTML)

2.- Bellani G, Laffey JG, Pham T, Fan E, Brochard L, Esteban A, et al. Epidemiology, patterns of care, and mortality for patients with acute respiratory distress síndrome in intensive Care Units in 50 Countries. JAMA 2016; 315(8):788-800. ( PubMed) (HTML)

3.- Fan E, Brodie D, Slutsky AS. Acute respiratoria distress síndrome. Advances in diagnosis and treatment. JAMA 2018;319(7): 698-710. (․ubMed) (TML)

4.- Amato MB, Barbas CS, Medeiros DM, Magaldi RB, Schettino GP, Lorenzi-Filho G, et al. Effect of a protective-ventilation strategy on mortality in the acute respiratory distress syndrome. N Engl J Med. 1998;338(6):347-54. ( $\underline{\text { PubMed }}$ (

5.- Amato MB, Meade MO, Slutsky AS, Brochard L, Costa EL, Schoenfeld DA, et al. Driving pressure and survival in the acute respiratory distress syndrome. $\mathrm{N}$ Engl $\mathrm{J}$ Med.2015;372(8):747-55. (ubMed) (ㄴML)

Correspondencia al autor

Isabel de la Calle Gil

isabel.calle.gil@gmail.com

MIR. Unidad de Cuidados Intensivos de Anestesia.

Hospital Universitario 12 de Octubre. Madrid.

Aceptado para el blog en marzo de 2019 